
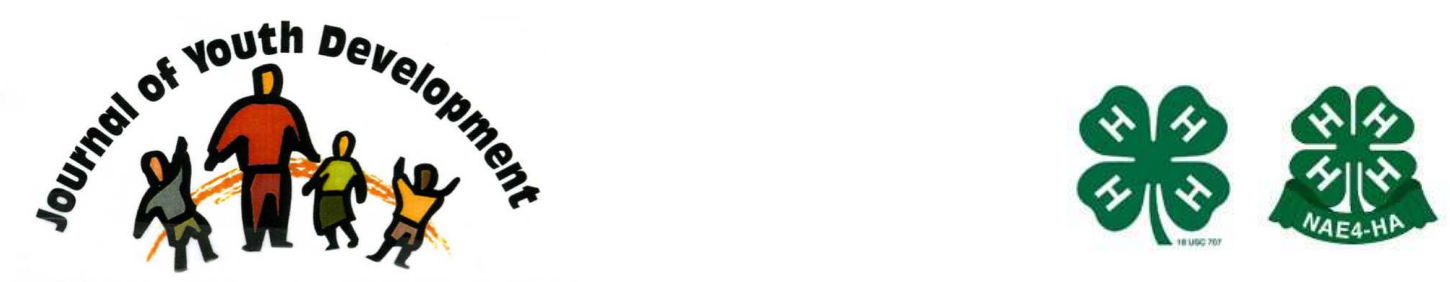

Bridging Research \& Practice

\title{
Measuring Physical Activity in Youth Settings: Considerations for Instrument Selection
}

Benjamin D. Hickerson

Department of Recreation, Park \& Tourism Management

The Pennsylvania State University

University Park PA

bdh14@psu.edu 


\title{
JOURNAL OF YOUTH DEVELOPMENT \\ bridging research and practice

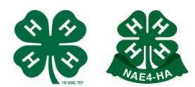

Bridging Research \& Practice

Volume 6, Number 4, Winter 2011

Article 110604RS001

\section{Measuring Physical Activity in Youth Settings: Considerations for Instrument Selection}

\author{
Benjamin D. Hickerson
}

\begin{abstract}
Increasing physical activity participation has become one of the primary strategies for prevention of early-onset health conditions including obesity and Type II diabetes. Youth programs including summer camps and after-school programs are premium providers of physical activity opportunities, but researchers and administrators of these programs must be able to effectively collect and interpret physical activity data to make program adjustments and communicate results. This article reviews existing methods for physical activity measurement including self-reports and objective instruments and makes suggestions for their applicability. Pedometers are covered in-depth as they may be the method of choice in many youth settings. These devices are unobtrusive, have a relatively low cost, and provide excellent data quality. Proper physical activity measurement in youth settings can provide information about effective intervention strategies and may also encourage on-site participants to increase their physical activity frequency.
\end{abstract}

\section{Introduction}

In the past two decades more attention has been directed toward child and adolescent health. Research evidence indicated that a number of poor health conditions including obesity and Type II diabetes were beginning to manifest at young ages. Prevalence of obesity in children aged 6 - 11 years old increased steadily beginning in 1965 with the greatest escalations following the mid-1980's (Troiano, Flegal, Kuczmarski, Campbell, \& Johnson, 1995). Data from the National Health and Nutrition Examination Survey indicated that 33\% of $6-11$ year old youth are currently overweight or at risk of being overweight (Ogden, Carroll, \& Flegal, 2008). Predictions for the current generation of youth project that they will be the most obese generation in United States' history and without interventions the next generation is likely to continue the trend (Hill \& Trowbridge, 1998).

One of the primary strategies for youth and adolescent obesity prevention is to increase physical activity participation. In 2005 The United States Department of Health and Human Services (USDHHS) boosted guidelines to recommend that youth under the age of 18 years old 
participate in at least 60 minutes of physical activity each day. However, a sizable percentage of youth in the United States do not partake in adequate amounts of physical activity (Troiano et al., 2008; Tudor-Locke, Johnson, \& Katmarzyk, 2010). Meeting these recommended physical activity guidelines can result in a host of health benefits including muscular strength, reduced blood pressure, and a healthy heart (Strong et al., 2005).

A promising area for increasing youth physical activity is within the places and programs offered by schools, public recreation agencies, nonprofit organizations, and other community services. Many offerings such as summer camps, after school programs, and neighborhood clubs are inherently rich with physical activity opportunities, but program leaders need tools to collect and assess data describing their participants. To foster physical activity awareness and participation, youth program coordinators have started to consider measuring and documenting physical activity occurring in their programs. Measurement of physical activity could help inform program decisions or provide positive information that could be reported to the public (e.g., $90 \%$ of participants in this program meet USDHHS guidelines).

A number of options exist to collect physical activity data including self-reports and objective measurement (e.g., pedometers). Each approach has different strengths and weaknesses, but certain constraints and needs may lead an investigator to choose one over the other. Therefore, the purpose of this paper is to guide the selection of practical instruments for monitoring physical activity in youth settings. The discussion will especially focus on pedometers, which offer an affordable option with the greatest utility for encouraging, measuring, and advertising physical activity.

\section{Self-reports of Physical Activity}

Prior to the invention of movement-sensing devices (e.g., pedometers), self-reports of physical activity were the most commonly used medium. Participants are typically provided with a survey or worksheet instructing them to rate their physical activity on a Likert-type scale or estimate their number of minutes of physical activity participation. Ideally this data is collected over short time intervals (e.g., one day) so participants have less difficulty recalling their behaviors. The positive characteristics of self-reports include their inexpensiveness and ease of collecting data from large groups.

Although they are accepted as valid measurement tools, physical activity self-report instruments have been criticized for subjectivity, recall errors, misinterpretations, and social desirability (Sirard \& Pate, 2001). The essence of a self-report relies "completely on a respondent's ability to provide good information about his or her own behaviors" (Matthews, 2002, p. 108). Although participants may try to provide their best estimates, physical activity participation is frequently overestimated in self-reports. Children especially have difficulty estimating their frequency of physical activity participation.

Depending upon the type of self-report instrument, the data may be too generic for practical interpretation. Likert-type instruments (e.g., strongly agree to strongly disagree) may be useful for comparing groups (e.g., male versus female physical activity), but they do not provide a count of physical activity that can be expressed in numbers (e.g., minutes). A recent systematic review suggests if a self-report instrument is chosen instead of more objective methods, the following are the most promising to use with children (Chinapaw, Mokkink, van Poppel, van Mechelen, \& Terwee, 2010): 
- Physical Activity Questionnaire for Older Children (Crocker, Bailey, Faulkner, Kowalski, \& McGrath, 1997)

- Girls Health Enrichment Multisite Study Activity Questionnaire (Treuth et al., 2003)

- Children's Leisure Activities Study Survey (Telford, Salmon, Jolley, \& Crawford, 2004)

- The Physical Activity Questionnaire for Parents and Teachers (Harro, 1997)

- Assessment of Young Children's Activity using Video Technology (Tremblay, Inman, \& Willms, 2001)

- Children's Physical Activity Questionnaire (McMurray, Harrell, Bradley, Webb, \& Goodman, 1998)

\section{Objective Measurement of Physical Activity}

To address the issues with self-reports of physical activity, focus has shifted to scientific instruments used to collect objective data. The most complex objective methods require precise physiological measurements of participants. For example, individuals can ingest a substance known as doubly labeled water, a radioactive isotope $\left({ }^{2} \mathrm{H}_{2} 180\right)$, and provide urine samples after at least three days of physical activity participation. Energy expenditure (i.e., physical activity) is then determined by the body's carbon dioxide production as it is observed in the urine sample (Schoeller et al., 1986). This is one of the most accurate method of measurement, but the intrusiveness and costs are impractical. These strengths and limitations are similar for other techniques including indirect calorimetry and heart-rate monitors.

A more common application of objective physical activity measurement is the use of a pedometer or accelerometer. Users of these devices typically wear a small box on the waistband of their clothing in line with the knee of their dominant leg. A difference between the pedometer and accelerometer is the way the device functions. Accelerometers contain an internal piezoelectric crystal that is compressed when the user is in motion. Body accelerations are detected by the instrument and more vigorous action is differentiated from less intense movements by the degree of crystal compression. The accelerometer constantly collects data capturing multi-directional movement intensity in periods as short as one second, commonly referred to as epochs. The final output provides caloric expenditure and describes the length and intensity (e.g., high, moderate, low) of the user's physical activity (Crouter, Schneider, \& Bassett, Jr., 2005). Accelerometers are an accurate measure of physical activity, but researchgrade devices are costly $(>\$ 100)$, obligatory analysis software is necessary, and the data are difficult to interpret without technical expertise (Rowlands \& Eston, 2005; Tudor-Locke \& Myers, 2001).

In comparison to accelerometers, pedometers provide a cheaper, more easily interpretable solution to physical activity measurement. Most pedometers contain a small, weighted internal arm mechanism suspended from a spring that moves up and down as a person moves. Each time a step is taken the arm moves down, touches a contact, and a step is counted. The final output from the pedometer is the "stepcount", a single number that indicates the activity level (i.e., steps taken) of the user.

\section{The Validity and Reliability of Physical Activity Data Collected Using Pedometers}

As the need for objective physical activity measurement has increased, pedometers have become a useful and popular measurement device (Sirard \& Pate, 2001). To ensure that 
pedometers are a functional instrument for physical activity data collection, researchers have conducted a breadth of studies to establish validity. Construct validity for pedometers was established through positive relationships with other fitness indicators including: a six-minute walk test $(r=.69)$, timed treadmill test $(r=.41)$, and estimated maximum oxygen uptake ( $r=.22)$; (Tudor-Locke, Williams, Reis, \& Pluto, 2002a). Convergent validity, or the degree to which the measurement agrees with other expected instruments, was established through positive correlations with accelerometers $(r=.86)$, time in observed activities $(r=.82)$, varying measures of energy expenditure $(r=.68)$, and self-reported physical activity $(r=.33)$; (TudorLocke, Williams, Reis, \& Pluto, 2002b).

Pedometer research has also been conducted to determine consistency, or reliability. Vincent and Sidman (2003) conducted a test where a machine shook a Yamax Digiwalker SW-200 pedometer to determine the accuracy of the device. This test yielded less than $3 \%$ error in actual shakes versus shakes recorded $(r=.97)$. This test was then repeated after seven weeks of pedometer use. No significant differences were found in the follow-up shake test, indicating that subsequent use of the device did not deteriorate its measurement accuracy.

However, not all pedometers are created equally. Pedometer units can range in price from $\$ 1$ to $\$ 100$ and their quality and features can impact the data they produce. Higher-end models of pedometers typically feature better springs (i.e., coil instead of hair) and can automatically reset themselves during long periods of data collection. Schneider, Crouter, and Bassett, Jr. (2004) suggest that "research-quality" pedometers should be purchased for the most accurate measurements. In their research they found that some models are more consistent than others (see Schneider et al.).

Recently a device known as a piezoelectric pedometer has become available, combining the low-cost of pedometers and the measurement quality of accelerometers (McClain \& TudorLocke, 2009). These instruments feature the piezoelectric crystal contained in an accelerometer, but have less features than a research-grade accelerometer. A piezoelectric pedometer can measure physical activity intensity, which is an upgrade to pedometers, but these devices are still reasonably expensive $(\sim 50)$ and the research about their reliability is still being developed.

Recently a device known as a piezoelectronic pedometer has become available, combining the low-cost of pedometers and the data quality of accelerometers (McClain \& Tudor-Locke, 2009). However, the research on these instruments is still being developed.

\section{The Relationship between Stepcount and Physical Activity}

To use and interpret the data collected by a pedometer, a researcher or program coordinator must first understand the "stepcount" collected by the device. For adults, 10,000 pedometercounted steps has gained acceptance as the threshold for reducing the risk of obesity and chronic disease caused by a lack of physical activity (Tudor-Locke \& Bassett, Jr., 2004). However, the USDHHS physical activity recommendation for adults is much lower than for children (i.e., 150 minutes per week vs. 60 minutes per day). Therefore it has been suggested that 10,000 steps per day are not enough for children to meet the recommended guidelines. Although children likely need more than 10,000 steps to achieve 60 minutes of moderatevigorous physical activity, no general consensus exists about the number of steps necessary.

Vincent and Pangrazi (2002a) collected four days of pedometer data from a large sample $(N=$ 711) of $6-12$ year old children and used mean values from the sample to determine that 
11,000 steps per day for girls and 13,000 steps per day for boys should be the standard. Two years later Tudor-Locke et al. (2004) used the same mean-based approach, but concluded from a larger $(N=1,954)$ international sample of $6-12$ year old children that the standards should be 12,000 steps per day for girls and 15,000 steps per day for boys.

These two sets of guidelines are the most commonly cited, but many have questioned the rationale behind their mean-based approach (Laurson et al., 2008). In a study where $11-15$ year old boys wore both pedometers and accelerometers, Jago et al. (2006) found that as few as 8,000 steps per day were enough to meet the 60-minute guidelines. Rowlands and Eston (2005) conducted a similar study with $8-10$ year old boys and girls and found that 12,000 steps per day for girls and 13,000 steps per day for boys resulted in more than 60 minutes of physical activity recorded by an accelerometer. Although no consensus has been reached, about $12,000-13,000$ steps counted on a reliable pedometer may be necessary to accrue 60 minutes of physical activity for youth under the age of 18 .

\section{Implementing and Using Pedometers in Youth Programs}

Pedometers have proven to be a cheap and accessible option for determining youth physical activity. At face value the stepcount can be useful, but there are a number of methods that can be used to provide additional insight into the raw physical activity numbers. To collect the most rigorous pedometer data certain standards must be followed, but any systematically collected pedometer data can be used to make practical assumptions for improvements to youth programming. Purchasing a high-quality research pedometer can increase the assurance of reliability, but there are other considerations including the time period of monitoring and data interpretation.

To make generalizations about physical activity occurring in a program, more than one day of data collection is necessary. Stepcounts could vary day-to-day, especially in programs like youth camps that may have different types of programming each day of the week. Research has determined that at least three to five days of monitoring are necessary to obtain a reliable measurement of physical activity (Trost, Pate, Freedson, Sallis, \& Taylor, 2000; Vincent \& Pangrazi, 2002b). Kang et al. (2009) reinforced that data are more accurate when there five or more days of data collection.

Some physical activity programming that takes place cannot be recorded by pedometers. The pedometer is placed on the body's vertical axis (i.e., waistband or hip) and therefore only ambulatory movements can be logged. Activities such as bicycling, weight training, swimming, rock-climbing, and horseback riding are not captured. To address this issue Miller, Brown, and Tudor-Locke (2006) created two simple conversion ratios that can be used to add steps to the pedometer count. The Simple Conversion Method (SIM) can be used to add 100 steps per minute for each minute of moderate physical activity (e.g., walking). The Intermediate Conversion Method (INT) can be used to add 200 steps per minute for each minute of vigorous physical activity (e.g., lap swimming).

Another concern with the use of pedometers is that the user will react to the device and attempt to be more active than usual. The magnitude of this concern depends on the purpose of the pedometer use. For research intended to generalize results and be prepared for publication in scientific journals, the researchers can restrict users from viewing their physical activity output by sealing the display on the device (Vincent \& Pangrazi, 2002b). The 
investigators may also choose to eliminate the first day of data collection because research suggests that reactivity deteriorates over time (Rowe, Mahar, Raedeke, \& Lore, 2004).

However, reactivity can have a positive effect for program administrators using pedometers to encourage physical activity. Pedometers can be given to participants and used to effectively set goals and increase physical activity (Tudor-Locke \& Lutes, 2009). This increase can amount to as many as $1,800-4,500$ additional steps per day (Richardson et al., 2008). Programmers can include a number of games to achieve these physical activity goals such as:

- Setting objectives for participants to increase their stepcount each day of the week for a set number of days (e.g., Day $1=5,000$ steps, Day $2=6,000$ steps).

- Creating teams randomly, based upon age groups, or other characteristics that battle to achieve the highest number of stepcounts in comparison to competing teams.

- Summing the totals from the participants in the program and plotting them on a map. For example, the group could set a goal to walk the distance of the length of the United States.

Researchers and practitioners may also look to evaluate the amount of physical activity in youth programming by comparing expected versus actual physical activity. The Physical Activities Compendium designed by Ainsworth et al. $(1993,2000)$ is a catalogue of research that has determined the intensity level of common physical activities. This instrument can be used to estimate expected amounts of energy expenditure associated with participation in a specific activity (e.g., softball, tennis, swimming). This number is known as the metabolic equivalency ratio (MET), which compares the ratio of work metabolic rate to a standard resting metabolic rate. More recently, a new Compendium has been developed exclusively for use with children (see Ridley, Ainsworth, \& Olds, 2008).

To make use of these compendiums, researchers can use the provided values to determine the expected amount of physical activity in a program and correlate this number with an objective measurement of participation (e.g., pedometer or accelerometer). For example, one would expect that programming with a higher number of expected METs would yield a larger stepcount. If researchers or practitioners found the opposite, that may indicate a need for programming re-evaluation.

\section{Conclusion}

Increasing physical activity participation has become a priority of public health interventions. Youth programs such as after school programs and summer camps have the potential to influence child and adolescent physical activity, but the administrators of these programs must first identify strategies and capacity for this important task. One of the first steps is to measure physical activity occurring in the programs. A number of methods for measuring physical activity exist, but some have greater limitations than others. Through a review of the associated literature, it seems that pedometers may be the most affordable and effective device for capturing physical activity data used for practical concerns. Information collected from these devices and other physical activity instruments can be used to influence physical activity and improve the quality of physical activity-based programs for youth in the United States.

\section{References}

Ainsworth, B.E., Haskell, W.L., Whitt, M.C., Irwin, M.L., Swartz, A.M., Strath, S.J., et al. (2000). Compendium of Physical Activities: An update of activity codes and MET intensities. Medicine \& Science in Sports \& Exercise, 32(9), S498-S516. 
Ainsworth, B.E., Haskell, W.L., Leon, A.S., Jacobs, D.R., Montoye, H.J., Sallis, J.F., et al. (1993). Compendium of Physical Activities: Classification of energy costs of human physical activities. Medicine \& Science in Sports \& Exercise, 25(1), 71-80.

Chinapaw, M.J.M., Mokkink, L.B., van Poppel, M.N.M., van Mechelen, W., \& Terwee, C. (2010). Physical activity questionnaires for youth: A systematic review of measurement properties. Sports Medicine, 40(7), 539-563.

Crocker, P.R.E., Bailey, D.A., Faulkner, R.A., Kowalski, K.C., \& McGrath, R. (1997). Measuring general levels of physical activity: Preliminary evidence for the Physical Activity Questionnaire for Older Children. Medicine \& Science in Sports \& Exercise, 29(10), 1344-1349.

Crouter, S.E., Schneider, P.L., \& Bassett, D.R., Jr. (2005). Spring-levered versus piezo-electric pedometer accuracy in overweight and obese adults. Medicine \& Science in Sports \& Exercise, $3710), 1673-1679$.

Harro, M. (1997). Validation of a questionnaire to assess physical activity of children ages 4-8 years. Research Quarterly for Exercise \& Sport, 68(4), 259-268.

Hill, J.O., \& Trowbridge, F.L. (1998). Childhood obesity: Future directions and research priorities. Pediatrics, 101(3), 570-574.

Laurson, K.R., Eisenmann, J.C., Welk, G.J., Wickel, E.E., Gentile, D.A., \& Walsh, D.A. (2008). Evaluation of youth pedometer-determined physical activity guidelines using receiver operator characteristic curves. Preventive Medicine, 46, 419-424.

Jago, R., Watson, K., Baranowski, T., Zakeri, I., Sunmi, Y., Baranowski, J., et al. (2006). Pedometer reliability, validity, and daily activity targets among 10- to 15 -year-old boys. Journal of Sport Sciences, 24, 241-251.

Kang, M., Bassett, D.R., Barreira, T.V., Tudor-Locke, C., Ainsworth, B., Reis, J.P., et al. (2009). How many days are enough? A study of 365 days of pedometer monitoring. Research Quarterly for Exercise and Sport, 80(3), 445-453.

Matthews, C.E. (2002). Use of self-report instruments to assess physical activity. In G. Welk (Ed.), Physical activity assessments for health-related research (pp. 107-123). Champaign, IL: Human Kinetics.

McClain, J.J., \& Tudor-Locke, C. (2009). Objective monitoring of physical activity in children: Considerations for instrument selection. Journal of Science and Medicine in Sport, 12(5), 526533.

McMurray, R.G., Harrell, J.S., Bradley, C.B., Webb, J.P., \& Goodman E.M. (1998). Comparison of a computerized physical activity recall with a tri-axial motion sensor in middle school youth. Medicine \& Science in Sports \& Exercise, 30(8), 1238-1245.

Miller, R., Brown, W., \& Tudor-Locke, C. (2006). But what about swimming and cycling? How to "count" non-ambulatory activity when using pedometers to assess physical activity. Journal of Physical Activity and Health, 3, 257-266. 
Ogden, C.L., Carroll, M.D., \& Flegal, K.M. (2008). High Body Mass Index for age among US children and adolescents, 2003-2006. Journal of The American Medical Association, 299(20), 2041-2045.

Richardson, C.R., Newton, T.L., Abraham, J.J., Sen, A., Jimbo, M., \& Swartz, A.M. (2008). A meta-analysis of pedometer-based walking interventions and weight loss. Annals of Family Medicine, 6(1), 69-77.

Ridley, K., Ainsworth, B.E., \& Olds, T.S. (2008). Development of a compendium of energy expenditures for youth. International Journal of Behavioral Nutrition and Physical Activity, 5(45).

Rowe, D.A., Mahar, M.I., Raedeke, T.D., \& Lore, J. (2004). Measuring physical activity in children with pedometers: Reliability, reactivity, and replacement of missing data. Pediatric Exercise Science, 16(4), 343-354.

Rowlands, A.V., \& Eston, R.G. (2005). Comparison of accelerometer and pedometer measures of physical activity in boys and girls, ages 8-10 years. Research Quarterly for Exercise and Sport, 76(3), 251-257.

Schneider, P.L., Crouter, S.E., \& Bassett, D.R., Jr. (2004). Pedometer measures of free-living physical activity: Comparison of 13 models. Medicine \& Science in Sports \& Exercise, 36(2), 331-335.

Schoeller, D.A., Ravussin, E., Schutz, Y., Acheson, K., Baertschi, P., \& Jequier, E. (1986). Energy expenditure by doubly labeled water: Validation in humans and proposed calculation. The American Journal of Physiology: Regulative, Integrative and Comparative Physiology, 250(19), R823-R830.

Sirard, J.R., \& Pate, R.R. (2001). Physical activity assessments in children and adolescents. Sports Medicine, 31(6), 439-454.

Strong, W.B., Malina, R.M., Blimkie, C.J., Daniels, S.R., Dishman, R.K., Gutin, B., et al. (2005). Evidence based physical activity for school-age youth. Journal of Pediatrics, 146, 732-737.

Telford, A., Salmon, J., Jolley, D., \& Crawford, D. (2004). Reliability and validity of physical activity questionnaires for children: The Children's Leisure Activities Study Survey (CLASS). Pediatric Exercise Science, 16, 64-78.

Tremblay, M.S., Inman, J.W., \& Willms, J.D. (2001). Preliminary evaluation of a video questionnaire to assess activity levels of children. Medicine \& Science in Sports \& Exercise, 33(12), 2139-2144.

Treuth, M., Sherwood, N.E., Butte, N.F., McClanahan, B., Obarzanek, E., Zhou, A., et al. (2003). Validity and reliability of activity measures in African-American girls for GEMS. Medicine \& Science in Sports \& Exercise, 35(3), 532-539.

Troiano, R.P., Flegal, K.M., Kuczmarski, R.J., Campbell, S.M., \& Johnson, C.L. (1995). Overweight prevalence and trends for children and adolescents. Archives of Pediatric Adolescent Medicine, 149, 1085-1091. 
Troiano, R.P., Berrigan, D., Dodd, K.W., Masse, L.C., Tilert, T., \& McDowell, M. (2008). Physical activity in the United States measured by accelerometer. Medicine \& Science in Sports \& Exercise, 40(1), 181-188.

Trost, S.G., Pate, R.R., Freedson, P.S., Sallis, J.F., \& Taylor, W.C. (2000). Using objective physical activity measures with youth: How many days of monitoring are needed? Medicine \& Science in Sports \& Exercise, 32(2), 426-431.

Tudor-Locke, C., \& Bassett, D.R., Jr. (2004). How many steps/day are enough? Preliminary pedometer indices for public health. Sports Medicine, 3, 41-48.

Tudor-Locke, C., Johnson, W.D., \& Katmarzyk, P.T. (2010). Accelerometer-determined steps per day in US children and youth. Medicine \& Science in Sports \& Exercise, 42(12), 2244-2250.

Tudor-Locke, C., \& Lutes, L. (2009). Why do pedometers work? A reflection upon the factors related to successfully increasing physical activity. Sports Medicine, 39(12), 891-993.

Tudor-Locke, C., \& Myers, A.M. (2001). Challenges and opportunities for measuring physical activity in sedentary adults. Sports Medicine, 31, 91-100.

Tudor-Locke, C., Pangrazi, R.P., Corbin, C.B., Rutherford, W.J., Vincent, S.D., Raustorp, A., et al. (2004). BMI-referenced standards for recommended pedometer-determined steps/day in children. Preventive Medicine, 38, 857-864.

Tudor-Locke, C., Williams, J.E., Reis, J.P., \& Pluto, D. (2002a). Utility of pedometers for assessing physical activity: Construct validity. Sports Medicine, 32(12), 281-291.

Tudor-Locke, C., Williams, J.E., Reis, J.P., \& Pluto, D. (2002b). Utility of pedometers for assessing physical activity: Convergent validity. Sports Medicine, 32(12), 795-808.

Vincent, S.D., \& Pangrazi, R.P. (2002a). An examination of the activity patterns of elementary school children. Pediatric Exercise Science, 14, 432-441.

Vincent, S.D., \& Pangrazi, R.P. (2002b). Does reactivity exist in children when measuring activity levels with pedometers? Pediatric Exercise Science, 14, 56-63.

Vincent, S.D., \& Sidman, C.L. (2003). Determining measurement error in digital pedometers. Measurement in Physical Education and Exercise Science, 7, 19-24.

(C) Copyright of Journal of Youth Development $~$ Bridging Research and Practice. Content may not be copied or emailed to multiple sites or posted to a listserv without copyright holder's express written permission. However, users may print, download or email articles for individual use. 